\title{
GENERATION OF AURORAL KILOMETRIC RADIATION IN A DIPOLE MAGNETIC FIELD: 3-D APPROACH
}

\author{
T. M. Burinskaya*, and M. M. Shevelev*
}

\begin{abstract}
We have investigated the propagation and amplification of Auroral Kilometric Radiation (AKR) in a narrow three-dimensional plasma cavity, in which a weakly relativistic electron beam propagates along the magnetic field. Both electron beam velocity components, parallel and perpendicular to the magnetic field are taken into account. Although the energy of transverse electron motion serves as a source of free energy for the development of electron cyclotron maser instability, it is found that for correct description of the AKR spectrum formation it is necessary to take the velocity component of electron motion directed along the magnetic field into account, because it gives a possibility for wave generation in a much wider frequency range. The results of calculations performed for thousands of waves have strengthened the assumption made in our previous paper [Burinskaya, 2013] on a basis of calculations for several waves, that the main factor, determining the wave energy at the time of wave escape from a source, is the duration of wave lifetime inside an amplification region. Thus, the global magnetic field inhomogeneity plays a key role in the formation of the AKR spectrum, because it defines for each wave its lifetime inside an amplification region and, by this means, the wave spectral intensity.
\end{abstract}

\section{Introduction}

Satellite measurements made above the auroral region during the last two decades have clearly shown that terrestrial Auroral Kilometric Radiation (AKR) sources are thin densitydepleted cavities extended along the magnetic field with a small latitudinal width about several tens of kilometers as compared to their longitudinal extent, which is of the order of 1000-2000 km. Plasma of AKR sources is tenuous with an electron population essentially composed of down-going, weakly relativistic particles [Louarn and Le Quéau, 1996; Mutel et al., 2008]. As downward-directed electrons encounter an increasing magnetic field at lower altitudes, their parallel energy is converted to perpendicular energy, resulting

* Space Research Institute, Russian Academy of Sciences, Moscow, Russia 
in the electron distribution with a positive perpendicular velocity gradient. Theoretical investigations have shown that distributions of this type are subject to the electron cyclotron maser instability which leads to the efficient energy transfer from electrons to electromagnetic waves [Wu and Lee, 1979]. The AKR is the most powerful nonthermal natural emission from Earth, but theoretical calculations of the wave amplification, performed without taking into account the wave propagation inside a source region in the presence of an inhomogeneous magnetic field, barely correspond to the observed AKR spectral intensity. Some calculations were made for several waves which had shown the necessity to take into account a global inhomogeneity of the magnetic field for the explanation of the AKR intensity [Zarka et al., 1986; Burinskaya, 2013]. Here we present the path-integrated growth rate calculations made for thousands of waves propagating inside a three-dimensional plasma cavity similar to the experimentally observed ones in a dipole magnetic field using the approximation of geometrical optics. In the present paper we explore the case when the initial ratio of parallel velocity of relativistic electrons to the speed of light is not equal zero (unlike in the previous papers) with a purpose to estimate the role of the longitudinal electron velocity in the AKR spectrum formation. Although the energy of transverse electron motion serves as a source of free energy for the development of electron cyclotron maser instability, it is found that the velocity of relativistic electrons along the magnetic field plays an important role in the formation of the AKR spectrum since it enables generation of waves in a broader frequency range.

\section{The model and basic equations}

We use a model of the AKR source in which the size of a density-depleted cavity along a fixed latitude is much larger than its transverse size, and cavity's side surfaces are aligned with the magnetic field lines. The distribution function of fast electrons, propagating inside the cavity and serving as a source of free energy for the development of electron cyclotron maser instability, is approximated by a ring like velocity distribution, $f\left(v_{\|}, v_{\perp}\right)=$ $\left(2 \pi v_{0 \perp}\right)^{-1} \delta\left(v_{\|}-v_{0}\right) \delta\left(v_{\perp}-v_{0 \perp}\right)$, where the subscripts $\|$ and $\perp$ correspond to velocity components parallel and perpendicular to the magnetic field, respectively. Although such a distribution function is idealized, the main characteristics of the unstable modes remain almost unchanged in comparison with more realistic distributions [Le Quéau and Louarn, 1989; Cairns et al., 2008]. The ions are assumed to be immobile and serve as a neutralizing background. Earth's magnetic field is described in terms of the dipole model in spherical coordinates $(r, \theta, \varphi)$ with a polar axis coinciding with the magnetic field axis and pointing upwards from Earth, the radius $r$ is measured from Earth's center and the azimuthal angle $\varphi$ is measured from the cavity center, having the coordinate $\varphi_{0}=0$. The central invariant magnetic latitude of the cavity is $\Lambda_{0}$. The density profile of the cold background plasma is taken as

$$
n_{\text {cold }}=\frac{n_{0}}{\left(r / 2 R_{E}\right)^{2}}\left[1-\exp \left(-\frac{\left(\Lambda-\Lambda_{0}\right)^{2}}{\sigma_{\Lambda}^{2}}-\frac{\left(\varphi-\varphi_{0}\right)^{2}}{\sigma_{\varphi}^{2}}\right)\right],
$$

and the density profile of weakly relativistic electrons, propagating inside the cavity, is

$$
n_{\text {hot }}=\frac{n_{0, h o t}}{\left(r / 2 R_{E}\right)^{2}} \exp \left(-\frac{\left(\Lambda-\Lambda_{0}\right)^{2}}{\sigma_{\Lambda}^{2}}-\frac{\left(\varphi-\varphi_{0}\right)^{2}}{\sigma_{\varphi}^{2}}\right) ;
$$


where $R_{E}$ is Earth's radius; $n_{0}$ is the density of the cold background plasma outside the cavity at $r=2 R_{E} ; n_{0, h o t}$ is the density of energetic electrons at the center of the cavity at $r=2 R_{E} ; \sigma_{\Lambda}$ and $\sigma_{\varphi}$ are the characteristic cavity widths over $\Lambda$ and $\varphi$, respectively.

The propagation of waves generated inside the cavity due to the development of the electron cyclotron maser instability is studied in the geometrical optics approximation under the assumption that the real part of the wave frequency $\omega$ remains unchanged and the imaginary part of the frequency $\gamma$ is much smaller than the real part. The corresponding equations are the following [Bernstein, 1975]:

$$
\begin{aligned}
& \frac{d \mathbf{R}}{d t}=-\frac{\partial D_{R}}{\partial \mathbf{k}} / \frac{\partial D_{R}}{\partial \omega} \\
& \frac{d \mathbf{k}}{d t}=\frac{\partial D_{R}}{\partial \mathbf{R}} / \frac{\partial D_{R}}{\partial \omega} .
\end{aligned}
$$

Here, $\mathbf{R}$ is the position vector of the wave packet, $\mathbf{k}$ is the wave vector, and $D_{R}$ is the real part of the dispersion relation. Plasma may be considered as locally homogeneous at each point along the wave trajectory, because in the auroral region the magnetic field and plasma parameters change on spatial scales that are much longer than the AKR wavelength. The AKR is a strongly polarized emission mainly propagating as extraordinary X-mode waves [Hanasz et al., 2000; Panchenko et al., 2008], so we are concerned with the $\mathrm{X}$-mode dispersion relation only:

$$
\left(\frac{\varepsilon_{1}-N_{\|}^{2}}{\varepsilon_{1}}-N_{\perp}^{2}\right)\left(\frac{\varepsilon_{1}^{2}-\varepsilon_{2}^{2}}{\varepsilon_{1}}-N_{\|}^{2}-N_{\perp}^{2}\right)=\frac{N_{\|}^{2} \varepsilon_{2}^{2}}{\varepsilon_{1}^{2}},
$$

where $N_{\|, \perp}=k_{\|, \perp} c / \omega, c$ is the speed of light, $\varepsilon_{1}$ and $\varepsilon_{2}$ are the components of the dielectric tensor, which can be represented as $\varepsilon_{1,2}=\varepsilon_{1,2(\text { hot })}+\varepsilon_{1,2(\text { cold })}$, because the waves travel in a medium with both relativistic and cold background electrons. Taking into account that in the space region under investigation the electron Langmuir frequency $\omega_{p}$ is much lower than the electron cyclotron frequency $\omega_{c}$, and the characteristic wavelength of the excited waves is much larger than the electron gyroradius, the following components of the dielectric tensor are found for the ring distribution:

$$
\begin{array}{ll}
\varepsilon_{1(\text { cold })}=1-\frac{\omega_{p(\text { cold })}^{2}(\mathbf{R})}{\omega^{2}-\omega_{c}^{2}(\mathbf{R})}, & \varepsilon_{2(\text { cold })}=\frac{\omega_{p(\text { cold })}^{2}(\mathbf{R}) \cdot \omega_{c}(\mathbf{R})}{\omega\left(\omega^{2}-\omega_{c}^{2}(\mathbf{R})\right)}, \\
\varepsilon_{1(\text { hot })}=1-\frac{\omega_{p(\text { hot })}^{2}(\mathbf{R})}{2 \omega^{2}}\left(F_{1}+F_{2}\right), & \varepsilon_{2(\text { hot })}=\frac{\omega_{p(h o t)}^{2}(\mathbf{R})}{2 \omega^{2}}\left(F_{1}-F_{2}\right)
\end{array}
$$

where $F_{1}$ and $F_{2}$ are

$$
\begin{aligned}
& F_{1}=\frac{\omega-k_{\|} v_{0}}{\left(\omega-\omega_{c}(\mathbf{R}) \cdot \Gamma-k_{\|} v_{0}\right)}+\frac{v_{0 \perp}^{2}\left(k_{\|}^{2} c^{2}-\omega \cdot \omega_{c}(\mathbf{R})\right)}{2 c^{2}\left(\omega-\omega_{c}(\mathbf{R}) \cdot \Gamma-k_{\|} v_{0}\right)^{2}}, \\
& F_{2}=\frac{\omega-k_{\|} v_{0}}{\left(\omega+\omega_{c}(\mathbf{R}) \cdot \Gamma-k_{\|} v_{0}\right)}+\frac{v_{0 \perp}^{2}\left(k_{\|}^{2} c^{2}-\omega \cdot \omega_{c}(\mathbf{R})\right)}{2 c^{2}\left(\omega+\omega_{c}(\mathbf{R}) \cdot \Gamma-k_{\|} v_{0}\right)^{2}} ;
\end{aligned}
$$

Here $\Gamma=\left(1-\left(v_{0 \perp}^{2}+v_{0}^{2}\right) / 2 c^{2}\right), \omega_{p(\text { cold })}$ and $\omega_{p(h o t)}$ are the local plasma frequencies determined by the densities of the cold background (Equation (1)) and weakly relativistic (Equation (2)) electrons, respectively. 
In the next section we present the results calculated for the initial parameters adapted to the AKR generation at an altitude $r \approx 2 R_{E}$, where the local electron gyrofrequency is $200 \mathrm{kHz}$, in a cavity with the central magnetic latitude $\Lambda=72^{\circ}$ corresponding to the L-shell 10.5. The parameters fall in the range of experimentally observed values [Louarn and Le Quéau, 1996]: $n_{0}=5 \mathrm{~cm}^{-3}, n_{0, h o t}=1 \mathrm{~cm}^{-3}$, and the transverse energy of weakly relativistic electrons is $4 \mathrm{keV}$. The cavity dimensions over $\Lambda$ and $\varphi$ are $\sigma_{\Lambda}=0.005$ and $\sigma_{\varphi}=0.23$, respectively. Although the source of free energy for the excitation of the cyclotron maser instability is the transverse energy of relativistic electrons, the role of the electron energy along the magnetic field may be very important in the formation of the AKR spectrum in inhomogeneous plasma. This is because the dispersion relation under consideration is modified when the electron velocity parallel to the magnetic field is taken into account [Burinskaya and Shevelev, 2016], and thus significant changes of wave trajectories inside a cavity are possible. We have made calculations for different values of the electron velocity component parallel to the magnetic field, $v_{0}$. The parallel and perpendicular velocity components change with altitudes in accordance with laws of the first adiabatic invariant and electron energy conservation.

\section{$3 \quad$ Results of calculations}

Using Equations (3) we have investigated the time history of thousands of wave packets launched from the center of the cavity (Equation (2)) at the altitude where the local cyclotron frequency is equal to $200 \mathrm{kHz}$. We consider waves with different initial values of the radial $k_{r}$ and azimuthal $k_{\varphi}$ components of the wave vector; the component $k_{\theta}$ is taken to be zero at the start point for all waves.

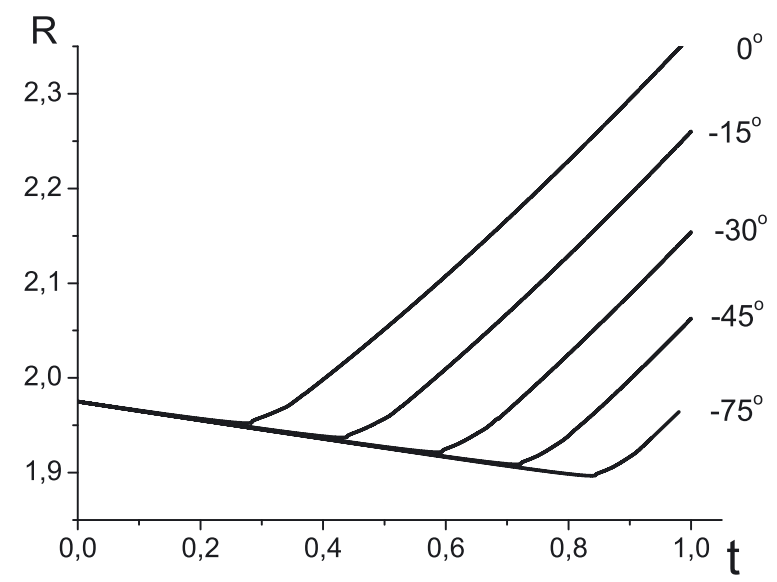

Figure 1: Normalized radial distance from Earth's center $R=r / R_{E}$ versus time normalized to $R_{E} / c$ for five wave packets with the same initial absolute value of the wave vector $|k|=0.8$ and different values of the angle $\phi$ between the wave vector and its azimuthal $\varphi$-component, shown on the right.

As an illustration, Figures 1, 2, 3 show the wave packet coordinates $(r, \theta, \varphi)$, respectively, 


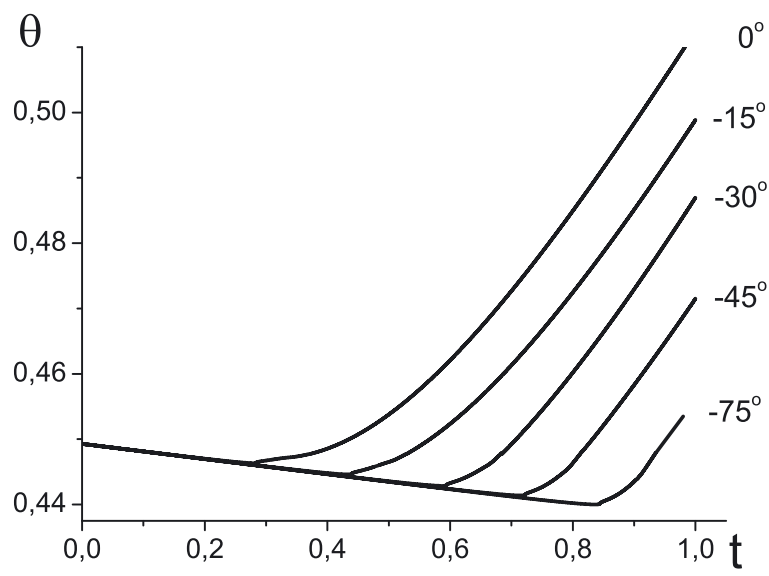

Figure 2: $\theta$ angle versus time normalized to $R_{E} / c$ for five wave packets with the same initial absolute value of the wave vector $|k|=0.8$ and different values of the angle $\phi$ between the wave vector and its azimuthal $\varphi$-component, shown on the right.

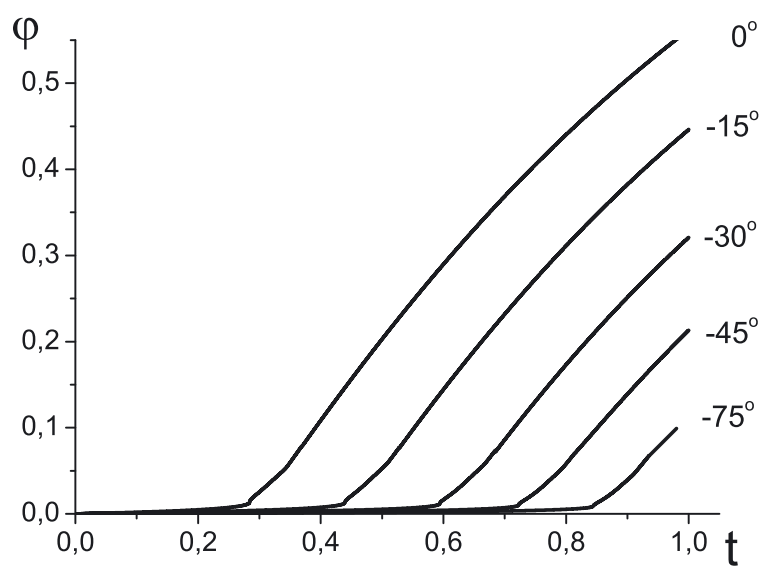

Figure 3: $\phi$ angle versus time normalized to $R_{E} / c$ for five wave packets with the same initial absolute value of the wave vector $|k|=0.8$ and different values of the angle $\phi$ between the wave vector and its azimuthal $\varphi$-component, shown on the right.

versus time normalized to $R_{E} / c$ for five different rays when $v_{0} / c=0.1$. In what follows, wave vectors are normalized to $\omega_{\text {Hfix }} / c$, and frequencies are normalized to $\omega_{\text {H fix }}\left(\omega_{H \text { fix }}\right.$ is the cyclotron frequency at the start position). In these figures we demonstrate rays launched with the same absolute value of the wave vector $|k|=0.8$ and different values of the angle $\phi$ between the wave vector and its azimuthal $\varphi$-component: $0^{\circ}\left(k_{r}=0, k_{\varphi}=0.8\right)$, $-15^{\circ}\left(k_{r}=-0.2, k_{\varphi}=0.77\right),-30^{\circ}\left(k_{r}=-0.4, k_{\varphi}=0.69\right),-45^{\circ}\left(k_{r}=-0.57, k_{\varphi}=0.57\right)$, $-75^{\circ}\left(k_{r}=-0.77, k_{\varphi}=0.2\right)$. The minus corresponds to wave propagation down to Earth. The normalized frequencies are $0.9895,1.0088,1.0282,1.0448$ and 1.0634, respectively. The frequencies of waves launched with $\phi=0^{\circ}$ and $\phi=-15^{\circ}$ are below the cutoff frequency of the background cold plasma calculated at $r=2 R_{E}$, that is 1.0099 . We 
found that a wave frequency increases with a growth of the radial wave vector component directed to Earth; and the higher the electron parallel velocity is, the more waves are generated with a frequency higher than the cutoff frequency of the background cold plasma at the altitude of their excitation. As follows from Figure 1, where $r=R_{E}$, all waves under consideration, including the wave starting with zero radial component $k_{r}=0$ (in contrast to the case when $v_{0}=0$ ), initially move towards Earth until a wave reaches a location where its radial group velocity becomes zero, afterwards the wave is reflected upward. Finally all five rays propagate inside the source upward from Earth until the local cutoff frequency of the surrounding plasma becomes equal or smaller than the wave frequency. Then rays leave the cavity and propagate outside the source region. Energy acquired by wave depends on its growth rate value along the ray trajectory and the duration of wave lifetime inside a source.
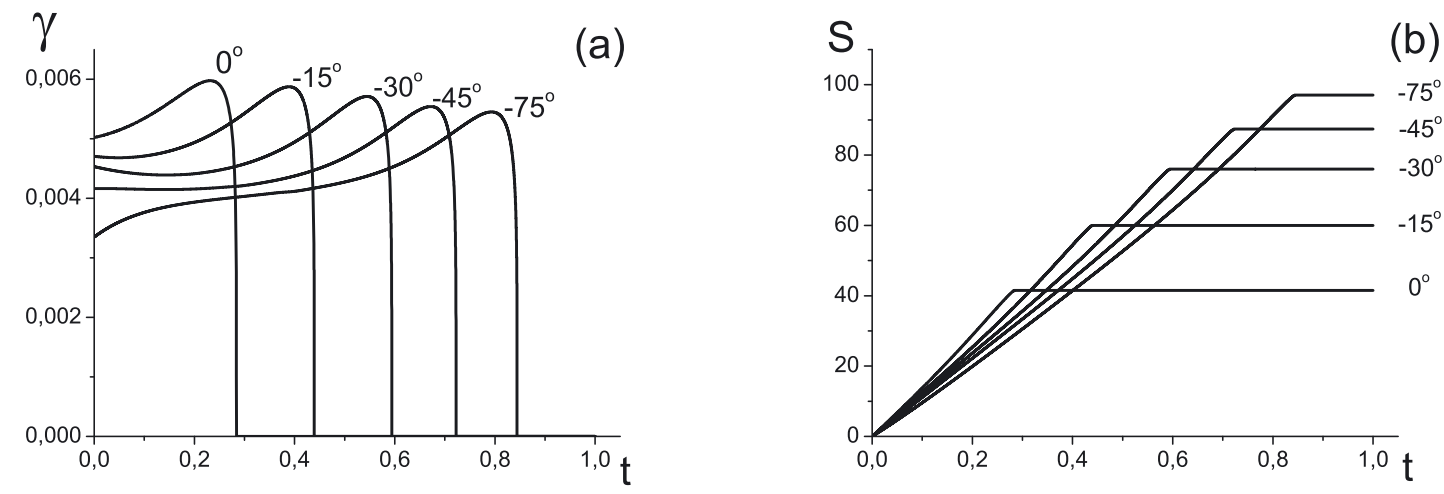

Figure 4: Growth rates normalized to $\omega_{H \text { fix }}$ along the trajectories versus dimensionless time (a) and amplification factors versus dimensionless time (b) of the same five wave packets shown in Figure 1.

For comparison of the influence of these two factors on the energy gained by a wave during its propagation inside a source region, we show the growth rate values along wave trajectories and amplification factors $S=\int_{0}^{t} \gamma(t) d t$ as functions of time in Figure 4(a) and Figure 4(b), respectively. An important point is that the wave $\left(\phi=-75^{\circ}\right)$, which accumulated the highest energy during its propagation inside the source region, has the lowest growth rate as at the start position, when $t=0$, so during its travel inside an amplification region; and vice versa: the wave $\left(\phi=0^{\circ}\right)$ excited perpendicular to the magnetic field and tangentially to the cavity boundaries with the maximum linear growth rate gains the lowest energy.

Numerical results obtained for thousands of waves with different initial values of wave vectors (not shown here because of paper size limit) strongly confirm that the most amplified waves are the waves generated with a reasonably large radial wave vector component initially directed towards Earth and conducting the longest lifetime inside a source, although their growth rates are less than growth rates of waves excited perpendicular to the magnetic field. The results of our calculations made for a variety of waves are summarized in Figure 5, where the wave amplification factor at the output of the cavity is shown versus the wave relative frequency $\delta \omega=\left(\omega-\omega_{H \text { fix }}\right) / \omega_{H \text { fix }}$ for several values of 


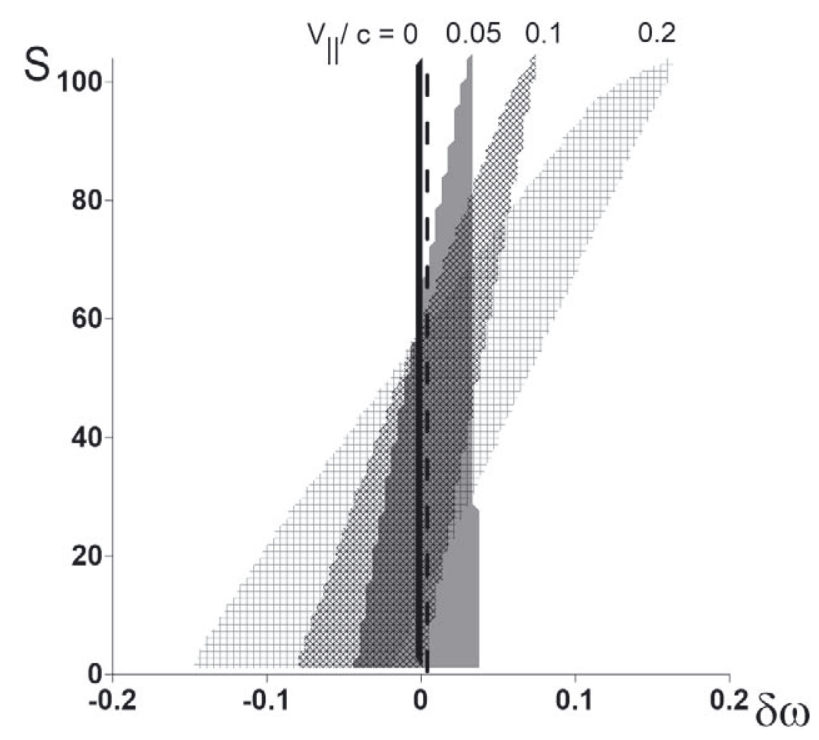

Figure 5: Wave amplification factors at the output from the source versus a relative frequency $\delta \omega=\left(\omega-\omega_{\text {H fix }}\right) / \omega_{\text {H fix }}$ for different values of the electron velocity component parallel to the magnetic field, shown on the top. For comparison, the narrow black strip shows the results obtained for $v_{0}=0$, and the vertical dashed line shows the cutoff frequency of the background plasma outside the cavity at the starting point.

the electron velocity parallel to the magnetic field denoted by different types of shades: $v_{0} / c=0.05,0.1,0.2$. For comparison the narrow black strip shows the results obtained for $v_{0}=0$, and the vertical dashed line shows the cutoff frequency of the background plasma outside the cavity at the starting point $\omega_{\text {cutoff }}$. It is clearly seen that for the case $v_{0} / c=0$ all waves are excited with frequencies below $\omega_{\text {cutoff }}$. When $v_{0} \neq 0$, the frequency range of excited waves grows substantially as $v_{0}$ increases, and the spectrum of waves with the largest amplification factors broaden in the frequency range above the cutoff frequency of the background plasma at the wave generation altitude.

\section{Conclusions}

Propagation and amplification of the Auroral Kilometric Radiation (AKR) in a narrow three-dimensional plasma cavity, in which a weakly relativistic electron beam propagates along the magnetic field, is investigated using the approximation of geometrical optics. A dipolar magnetic field model is adopted in our study. Both electron beam velocity components, parallel and perpendicular to the magnetic field, varying with altitudes in accordance with the laws of the first adiabatic invariant and electron energy conservation, are taken into account. The results of calculations performed for thousands of waves have strengthened the assumption, made on a basis of calculations for several waves, that the main factor, determining the wave energy at the time of wave escape from a source, is the duration of wave lifetime inside an amplification region. Thus, the global magnetic field 
inhomogeneity plays a key role in the formation of the AKR spectrum, because it defines for each wave its lifetime inside an amplification region and, by this means, the wave spectral intensity. It is found that the component of fast electron velocity parallel to the magnetic field enables generation of waves in a broader frequency range, and waves with the largest amplification factors at the output of the cavity have frequencies exceeding the cutoff frequency of the background plasma at the wave generation altitude.

Acknowledgments. This study was supported partially by a grant of RFBR 16-02-00079 and partially by a grant of RFBR 16-52-16010. The authors are also thankful to Michel Parrot for useful discussions within the framework of the RFBR grant 16-52-16010. The Editors thank Sébastien Hess and one anonymous reviewer for their help in evaluating this paper.

\section{References}

Bernstein, I. B., Geometric optics in space- and time-varying plasmas, Phys. Fluids, 18, 320-324, 1975.

Burinskaya, T. M., Ray tracing model of the auroral kilometric radiation in the 3-D plasma cavity, Ann. Geophys., 31, 1645-1652, 2013.

Burinskaya, T. M. and M. M. Shevelev, Generation of auroral kilometric radiation by a finite-size source in a dipole magnetic field, Plasma Phys. Rep., 42, 929-935, 2016.

Cairns, R. A., I. Vorgul, and R. Bingha, Cyclotron maser radiation from an inhomogeneous plasma, Phys. Rev. Lett., 101, id.215003, 2008.

Hanasz, J., M. Y. Boudjada, R. Schreiber, Z. Krawczyk, M. Malycha, M. M. Mogilevsky, H. O. Rucker, and T. V. Romantsova, Dynamic spectra of the Stokes parameters for the dayside and nightside Auroral Kilometric Radiation, Geophys. Res. Lett., 27, 1631-1634, 2000.

Le Quéau, D., and P. Louarn, Analytical study of the relativistic dispersion: Application to the generation of the auroral kilometric radiation, J. Geophys. Res., 94, 26052616, 1989.

Louarn, P., and D. Le Quéau, Generation of the Auroral Kilometric Radiation in plasma cavities: - I. Experimental study, Planet. Space Sci., 44, 199-210, 1996.

Mutel, R. L., I. W. Christopher, and J. S. Pickett, Cluster multispacecraft determination of AKR angular beaming, Geophys. Res. Lett., 35, L07104, 2008.

Panchenko, M., J. Hanasz, and H. O. Rucker, Estimation of linear wave polarization of the auroral kilometric radiation, Radio Sci., 43, RS1006, 2008.

Wu, C.S., and L.C. Lee, A theory of terrestrial kilometric radiation, Astrophys. J., 230, 621-626, 1979.

Zarka, P., D. Le Quéau, and F. Genova, The Maser Synchrotron Instability in an inhomogeneous medium: Determination of the spectral intensity of Auroral Kilometric Radiation, J. Geophys. Res., 91, 13542-13558, 1986. 